\title{
Relation between surgeon age and postoperative outcomes: a population-based cohort study
}

\author{
Raj Satkunasivam MD MS, Zachary Klaassen MD MSc, Bheeshma Ravi MD PhD, Kai-Ho Fok MD, \\ Terri Menser MBA PhD, Bita Kash MBA PhD, Brian J. Miles MD, Barbara Bass MD, Allan S. Detsky MD PhD CM, \\ Christopher J.D. Wallis MD PhD
}

Cite as: CMAJ 2020 April 14;192:E385-92. doi: 10.1503/cmaj.190820

\begin{abstract}
BACKGROUND: Aging may detrimentally affect cognitive and motor function. However, age is also associated with experience, and how these factors interplay and affect outcomes following surgery is unclear. We sought to evaluate the effect of surgeon age on postoperative outcomes in patients undergoing common surgical procedures.
\end{abstract}

METHODS: We performed a retrospective cohort study of patients undergoing 1 of 25 common surgical procedures in Ontario, Canada, from 2007 to 2015. We evaluated the association between surgeon age and a composite outcome of death, readmission and complications.
We used generalized estimating equations for analysis, accounting for relevant patient-, procedure-, surgeon- and hospital-level factors.

RESULTS: We found 1159676 eligible patients who were treated by 3314 surgeons and ranged in age from 27 to 81 years. Modelled as a continuous variable, a 10-year increase in surgeon age was associated with a $5 \%$ relative decreased odds of the composite outcome (adjusted odds ratio [OR] 0.95, 95\% confidence interval [CI] 0.92 to $0.98, p=0.002$ ). Considered dichotomously, patients receiving treatment from surgeons who were older than
65 years of age had a $7 \%$ lower odds of adverse outcomes (adjusted OR 0.93, $95 \% \mathrm{Cl} 0.88-0.97, p=0.03$; crude absolute difference $=3.1 \%$ ).

INTERPRETATION: We found that increasing surgeon age was associated with decreasing rates of postoperative death, readmission and complications in a nearly linear fashion after accounting for patient-, procedure-, surgeon- and hospital-level factors. Further evaluation of the mechanisms underlying these findings may help to improve patient safety and outcomes, and inform policy about maintenance of certification and retirement age for surgeons.
$\mathrm{H}$

igh-quality surgical outcomes require sound knowledge, communication skills, clinical judgment and technical proficiency. ${ }^{1}$ Aging of the surgical workforce has been well recognized; however, the effect on patient outcomes is unclear. ${ }^{2,3}$ The effect of aging, namely cognitive impairment, decline in visual acuity and worsening motor function, may negatively affect surgical ability. ${ }^{4}$ However, age-related decline may be offset by the older surgeon's years of clinical acumen, surgical experience and clinical judgement. In addition, because surgeon volume is associated with improved surgical outcomes, ${ }^{5,6}$ it is unclear whether surgical volume may offset age-related effects. Thus, surgical volume and age-related effects need to be evaluated concurrently. ${ }^{7,8}$

Published evidence about the effect of physician age and experience on surgical outcomes shows mixed results. ${ }^{9-11}$ Among Medicare beneficiaries undergoing pancreatectomy, coronary artery bypass grafting and carotid endarterectomy, mortality was higher among those treated by surgeons older than 60 years, especially those with low volumes. ${ }^{12}$ This was corroborated in subsequent studies of particular surgical procedures. ${ }^{13-15}$ In 2018, an observational study involving patients in the United States who were Medicare beneficiaries reported that patients who were treated by older surgeons had lower rates of mortality than those treated by younger surgeons. ${ }^{16}$ However, these data lacked generalizability because the study included only a limited number of nonelective surgical procedures in patients 65 years and older and failed to inform the safety of older physicians performing most surgeries, elective procedures. We aimed to fill these knowledge gaps by assessing the association between surgeon age and outcomes across a broad range of patient age, surgical specialties and common procedures, while adjusting for surgeon volume. 


\section{Methods}

\section{Study population and setting}

We conducted a population-based, retrospective cohort study of adult patients undergoing common surgical procedures in Ontario between Jan. 1, 2007, and Dec. 31, 2015, to evaluate the association between surgeon age and 30-day postoperative outcomes. Eligible residents of Ontario receive insurance for physician and hospital services through a single government payer, the Ontario Health Insurance Program (OHIP). We included patients who underwent 1 of 25 common procedures performed by surgeons in a variety of subspecialties to ensure generalizability, including both open and laparoscopic approaches when relevant. ${ }^{17}$ We engaged in multidisciplinary consultation (i.e., consensus discussion involving internal medicine, general surgery, urology and orthopedic surgery) to select the procedures. This study was reported according to Strengthening the Reporting of Observational Studies in Epidemiology (STROBE) guidelines ${ }^{18}$ and Reporting of studies Conducted using Observational Routinely-collected health Data (RECORD) statement. ${ }^{19}$

\section{Data sources}

We linked the following data sets using encrypted patient identifiers: the OHIP database (which tracks claims paid for physician billings, laboratories and, for determination of postoperative outcomes, all OHIP-funded medical interventions ${ }^{20}$ ); the Canadian Institute for Health Information (CIHI) Discharge Abstract Database (DAD; which contains records for admissions to hospita ${ }^{121}$ ); the $\mathrm{CIHI}$ National Ambulatory Care Reporting System (which contains records for visits to the emergency department); the Registered Persons Database for demographic information and determination of death;22 and the Corporate Provider Database for surgeon-level data. We identified surgical procedures using OHIP fee codes.

\section{Outcomes}

Our composite primary outcome was the presence of any of death, complications or readmission (to any hospital in the province of Ontario) in the 30 days after surgery. ${ }^{23}$ We used a previously defined measure of surgical complications associated with major morbidity: acute renal failure, bleeding requiring transfusion of 4 or more units within the first 72 hours after surgery, cardiac arrest requiring cardiopulmonary resuscitation, coma with a duration of 24 hours or more, deep venous thrombosis, myocardial infarction, ventilator use for 48 hours or more, pneumonia, pulmonary embolism, stroke, major disruption of wound, infection of surgical site, sepsis, septic shock, systemic inflammatory response syndrome, unplanned return to the operating room, vascular graft failure and shock. ${ }^{23}$ These outcomes were ascertained from health administrative data using a combination of uniformly collected procedural and diagnostic codes for all hospitals and all patients in Ontario. ${ }^{23,24}$ Our secondary outcomes were individual components of the composite outcome and hospital length of stay (LOS).

We identified surgeon age using linked administrative databases. A priori, we operationalized age as a continuous variable, as well as in increments of 10 years ( $<40,40-49,50-59,60-69$ and $\geq 70 \mathrm{yr}$ ), and as a dichotomous variable, using younger than 65 years versus 65 years or older, because this is considered the standard retirement age in the general workforce and when some surgeons plan to stop operating. 25

We classified surgical procedures as emergent or elective using the admission variables from the CIHI-DAD database. We considered all same-day or outpatient surgery procedures to be elective. We obtained patient age and sex, geographic location (Local Health Integration Networks), ${ }^{26}$ geographically derived socioeconomic status, rurality and general comorbidity (Johns Hopkins Aggregated Diagnosis Group, ADG) ${ }^{27}$. We also collected data for sex of the surgeon, years in practice, specialty and surgical volume. We determined surgical volume for each specific procedure by identifying the number of such procedures the operating surgeon performed in the previous year, operationalized in quartiles among the study cohort. We used identifiers for hospital institutions to account for facility-level variability.

\section{Statistical analysis}

We used descriptive statistics to compare the characteristics of patients and surgeons by surgeon age groups using standardized differences, ${ }^{28}$ with a clinically important difference defined as greater than $0.10 .^{28}$

We explored the relation between surgeon age and 30-day outcomes by plotting adjusted complication rate per 1000 by surgeon age. Because there were few surgeons who contributed to outcomes at the extremes of age, we restricted this analysis to ages 29-80 years and grouped surgeon age into increments of 2 years for the purposes of locally weighted scatterplot smoothing. These data were plotted with 95\% confidence intervals (Cls) and fitted with a locally weighted scatterplot smoothing line to depict the overall trend. In addition, we used adjusted restricted cubic splines with 5 knots to model the relation between surgeon age and 30-day outcomes.

We used multivariable generalized estimating equations with a compound symmetry correlation structure with a logit link to estimate the association between surgeon age and outcomes, accounting for patient-, surgeon- and hospital-level differences. We used average values for all variables (means for continuous variables and proportions for categorical variables) to estimate predicted probabilities. We performed these analyses with 3 different operationalizations of surgeon age: continuous, categorical by decade and dichotomized around 65 years. We scaled the continuous variable to report 10-year increments to provide a more comprehensible effect estimate. Because years in practice and surgeon age are highly collinear, we did not include the former variable. To examine the association between surgeon age and LOS, we used a similar approach that employed Poisson regression. The unit of analysis was the patient. We accounted for clustering at the level of the hospital or facility with generalized estimating equation models accounting for correlation in outcomes based on procedural fee codes.

In addition to surgeon age, we considered a separate model using years in practice as the exposure variable of interest in its place. Using the model of dichotomized surgeon age, we performed stratified analyses according to patient-, physician- and hospital-level characteristics to explore interactions between these factors and the association between surgeon age and postoperative outcomes. 
Table 1 (part 1 of 2): Baseline characteristics of the study cohort population who had surgery* in Ontario from Jan. 1, 2007 , to Dec. 31, 2015, stratified by surgeon age

\begin{tabular}{|c|c|c|c|}
\hline \multirow[b]{2}{*}{ Characteristic } & \multicolumn{2}{|c|}{ No. $(\%)$ of patients $\dagger$} & \multirow[b]{2}{*}{$\begin{array}{l}\text { Standardized } \\
\text { difference }\end{array}$} \\
\hline & $\begin{array}{c}\text { Surgeon age } \\
(<65 \mathrm{yr}) \\
n=1090243\end{array}$ & $\begin{array}{c}\text { Surgeon age } \\
(\geq 65 \mathrm{yr}) \\
n=69433\end{array}$ & \\
\hline \multicolumn{4}{|l|}{ Patient } \\
\hline \multicolumn{4}{|l|}{ Age, yr } \\
\hline Mean \pm SD & $58.80 \pm 17.32$ & $59.51 \pm 16.50$ & 0.04 \\
\hline Median (IQR) & $60(47-72)$ & $61(48-72)$ & 0.04 \\
\hline \multicolumn{4}{|l|}{ Sex } \\
\hline Female & $654432(60.0)$ & $41312(59.5)$ & 0.01 \\
\hline Male & $435811(40.0)$ & $28121(40.5)$ & 0.01 \\
\hline \multicolumn{4}{|l|}{ Income quintile } \\
\hline 1 (lowest) & 203357 (18.7) & $13620(19.6)$ & 0.02 \\
\hline 2 & 216626 (19.9) & $14136(20.4)$ & 0.01 \\
\hline 3 & $217754(20.0)$ & $13846(19.9)$ & 0 \\
\hline 4 & $228148(20.9)$ & 13851 (19.9) & 0.02 \\
\hline 5 (highest) & $219653(20.1)$ & $13672(19.7)$ & 0.01 \\
\hline Missing & $4705(0.4)$ & $308(0.4)$ & 0 \\
\hline \multicolumn{4}{|l|}{ Rurality $\ddagger$} \\
\hline Urban & $926799(85.0)$ & $57328(82.6)$ & 0.07 \\
\hline Rural & $162506(14.9)$ & 12054 (17.4) & 0.07 \\
\hline Missing & $938(0.1)$ & $51(0.1)$ & 0 \\
\hline \multicolumn{4}{|l|}{ ADG category } \\
\hline $0-5$ & $291564(26.7)$ & $18562(26.7)$ & 0 \\
\hline $6-7$ & $262557(24.1)$ & $17064(24.6)$ & 0.01 \\
\hline $8-10$ & 326048 (29.9) & $21075(30.4)$ & 0.01 \\
\hline$\geq 11$ & 210074 (19.3) & $12732(18.3)$ & 0.02 \\
\hline \multicolumn{4}{|l|}{ Year of surgery } \\
\hline 2007 & $135552(12.4)$ & $7113(10.2)$ & 0.07 \\
\hline 2008 & 129867 (11.9) & $6673(9.6)$ & 0.07 \\
\hline 2009 & $125763(11.5)$ & $7213(10.4)$ & 0.04 \\
\hline 2010 & $120517(11.1)$ & 7544 (10.9) & 0.01 \\
\hline 2011 & $119427(11.0)$ & $7531(10.8)$ & 0 \\
\hline 2012 & $116606(10.7)$ & $7678(11.1)$ & 0.01 \\
\hline 2013 & $117000(10.7)$ & 7937 (11.4) & 0.02 \\
\hline 2014 & $113908(10.4)$ & 8414 (12.1) & 0.05 \\
\hline 2015 & $111603(10.2)$ & 9330 (13.4) & 0.1 \\
\hline \multicolumn{4}{|l|}{ Physician } \\
\hline \multicolumn{4}{|l|}{ Physician sex } \\
\hline Female & 142869 (13.1) & $1250(1.8)$ & 0.44 \\
\hline Male & $947374(86.9)$ & $68183(98.2)$ & 0.44 \\
\hline \multicolumn{4}{|l|}{ Years in practice } \\
\hline Mean \pm SD & $14.24 \pm 8.18$ & $25.10 \pm 5.21$ & 1.58 \\
\hline Median (IQR) & $15(7-21)$ & $24(21-28)$ & 1.57 \\
\hline
\end{tabular}

Table 1 (part 2 of 2): Baseline characteristics of the study cohort population who had surgery* in Ontario from Jan. 1, 2007 , to Dec. 31, 2015, stratified by surgeon age

\begin{tabular}{|c|c|c|c|}
\hline \multirow[b]{2}{*}{ Characteristic } & \multicolumn{2}{|c|}{ No. (\%) of patients $†$} & \multirow[b]{2}{*}{$\begin{array}{c}\text { Standardized } \\
\text { difference }\end{array}$} \\
\hline & $\begin{array}{c}\text { Surgeon age } \\
\text { (<65 yr) } \\
n=1090243\end{array}$ & $\begin{array}{c}\text { Surgeon age } \\
\begin{array}{c}(\geq 65 \mathrm{yr}) \\
n=69433\end{array}\end{array}$ & \\
\hline \multicolumn{4}{|c|}{ Surgical volume (quartiles) } \\
\hline 1 (lowest) & $269844(24.8)$ & $15233(21.9)$ & 0.07 \\
\hline 2 & $283846(26.0)$ & $15965(23.0)$ & 0.07 \\
\hline 3 & $269036(24.7)$ & $17678(25.5)$ & 0.02 \\
\hline 4 (highest) & $267517(24.5)$ & $20557(29.6)$ & 0.11 \\
\hline \multicolumn{4}{|l|}{ Specialty } \\
\hline $\begin{array}{l}\text { Cardiothoracic } \\
\text { surgery }\end{array}$ & $65838(6.0)$ & $2921(4.2)$ & 0.08 \\
\hline General surgery & $367612(33.7)$ & $17112(24.6)$ & 0.2 \\
\hline Neurosurgery & $41851(3.8)$ & $4781(6.9)$ & 0.14 \\
\hline $\begin{array}{l}\text { Obstetrics and } \\
\text { gynecology }\end{array}$ & $125775(11.5)$ & $9167(13.2)$ & 0.05 \\
\hline $\begin{array}{l}\text { Orthopedic } \\
\text { surgery }\end{array}$ & $309452(28.4)$ & $21581(31.1)$ & 0.06 \\
\hline Otolaryngology & $24373(2.2)$ & $2035(2.9)$ & 0.04 \\
\hline Plastic surgery & $61379(5.6)$ & $5463(7.9)$ & 0.09 \\
\hline Thoracic surgery & $12092(1.1)$ & $61(0.1)$ & 0.13 \\
\hline Urology & $78085(7.2)$ & $6233(9.0)$ & 0.07 \\
\hline Vascular surgery & $3786(0.3)$ & $79(0.1)$ & 0.05 \\
\hline \multicolumn{4}{|l|}{ Hospital } \\
\hline \multicolumn{4}{|l|}{ Practice setting } \\
\hline Academic & 369654 (33.9) & $29174(42.0)$ & 0.17 \\
\hline Community & $720589(66.1)$ & $40259(58.0)$ & 0.17 \\
\hline
\end{tabular}

Note: $A D G=$ Aggregated Diagnosis Group, $I Q R=$ interquartile range, $S D=$ standard deviation *Procedures included coronary artery bypass grafting, femoral-popliteal bypass, abdominal aortic aneurysm repair, appendectomy, cholecystectomy, gastric bypass, colon resection, liver resection, hysterectomy, anterior or posterior spinal decompression, anterior or posterior spinal arthrodesis, craniotomy for brain tumour, total knee replacement, total hip replacement, open repair of femoral neck or shaft fracture, total thyroidectomy, neck dissection, lung resection, radical cystectomy, radical prostatectomy, transurethral resection of prostate, carpal tunnel release, and breast reduction. †Unless specified otherwise.

‡Defined using standard ICES methodology that relies on residential postal codes.

We conducted a preplanned stratified analysis based on whether the surgical procedure was elective or emergent; the latter is a situation wherein the selection of the surgeon by the patient and referring physician is minimized. Finally, we considered post hoc stratified analyses considering case complexity (low v. high complexity) and era of surgery (2007-2010 v. 2013-2015).

Statistical significance was set at $p<0.05$ based on a 2-tailed comparison. All analyses were performed using SAS Enterprise Guide 6.1 (SAS Institute).

\section{Ethics approval}

This study was approved by the Sunnybrook Health Sciences Centre Research Ethics Board (385-2016). 


\section{Results}

We identified all patients who underwent 1 of the 25 index procedures during the study interval $(n=1534592)$. We excluded patients who were treated by physicians whose primary declared specialty was nonsurgical $(n=8753)$ and those who were younger than 18 years of age $(n=29158)$. We also excluded repeat procedures ( $n=282399$ ); each patient was included in the cohort only once. We excluded patients where the date of death preceded the date of surgery in the database $(n=332)$. Finally, to capture data on hospital-level factors, we excluded patients for whom the treating institution could not be identified ( $n=54263)$.

These patients were treated over the study interval by 3314 surgeons, ranging in age from 27 to 81 years. Of these eligible patients, $234293(20.3 \%)$ were treated by surgeons who were younger than 40 years of age, whereas 405769 (35.0\%), 340213 (29.3\%), 162403 $(14.0 \%)$ and $15998(1.4 \%)$ patients were treated by surgeons aged 40-49, 50-59, 60-69 and 70 years or older, respectively (Appendix 1, Supplemental Table 1, available at www.cmaj.ca/lookup/suppl/ doi:10.1503/cmaj.190820/-/DC1). Older surgeons ( $\geq 65 \mathrm{yr}$ ) were more likely to be in the highest quartile for surgical volume and to practice in an academic setting (Table 1). Older surgeons performed proportionally more procedures in neurosurgery and fewer procedures in general and thoracic surgery (Table 1 ).

A

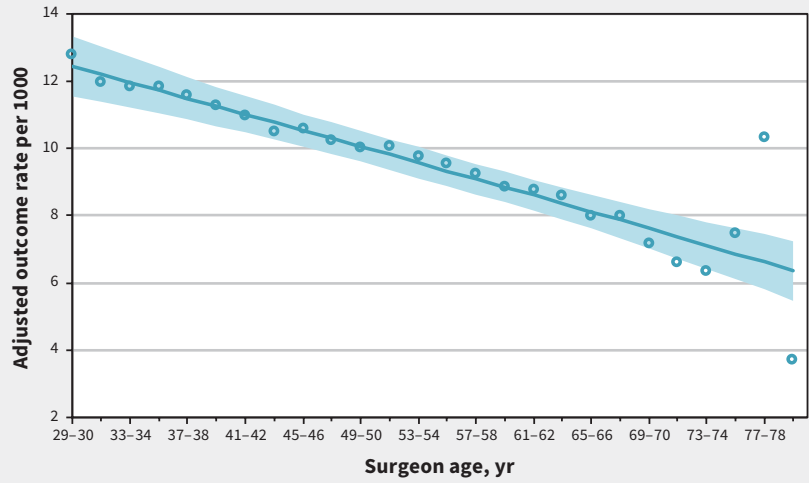

C

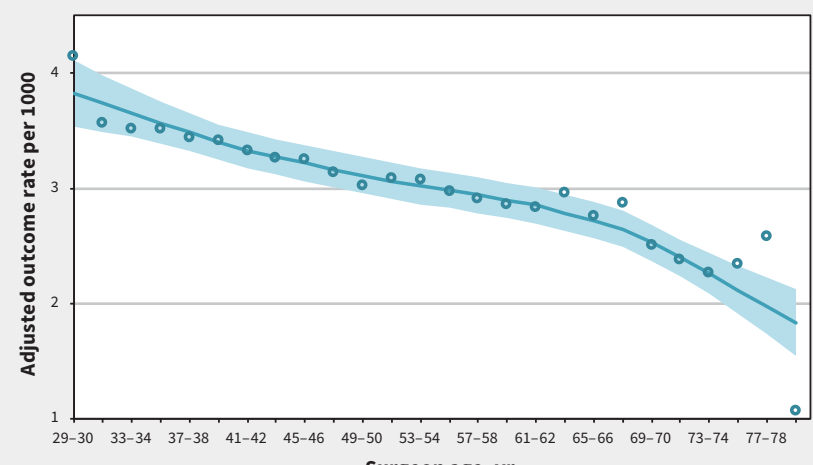

Surgeon age, yr
Overall, 138191 of 1159676 (11.9\%) patients experienced a composite outcome. We graphically explored the relation between 30-day postoperative outcomes and surgeon age, adjusting for patient-, procedure-, surgeon- and hospital-level factors. As shown in Figure 1, rates of the composite primary outcome and of each of the individual secondary outcomes showed an inverse relation to surgeon age. Restricted cubic splines evaluating the relation between surgeon age and each outcome showed a similar linear relation (Appendix 2, available at www. cmaj.ca/lookup/suppl/doi:10.1503/cmaj.190820/-/DC1).

Operationalized continuously, each 10 -year increase in surgeon age was associated with a $5 \%$ decreased odds of the composite outcome (adjusted odds ratio [OR] 0.95, 95\% Cl 0.92-0.98), decreased odds of death (adjusted OR 0.95, 95\% Cl 0.91-0.998), hospital readmission (adjusted OR $0.98,95 \% \mathrm{Cl} 0.97-0.99)$ and complications (adjusted OR 0.94, 95\% Cl 0.89-0.98) (Table 2). We did not observe a significant difference in the LOS according to surgeon age (adjusted rate ratio [RR] 1.01, 95\% Cl 0.99-1.02). We observed similar findings when surgeon age was operationalized categorically (data not shown). In a separate model, we evaluated years in practice in place of surgeon age and found similar results (adjusted OR 0.94 per decade, 95\% Cl 0.91-0.98).

Examined dichotomously, patients treated by older surgeons ( $\geq 65 \mathrm{yr}$ ) were significantly less likely to have the composite outcome

B

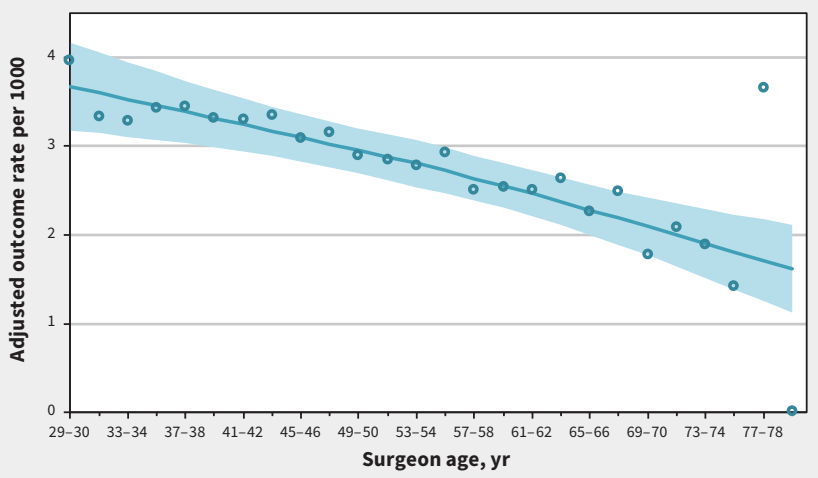

D

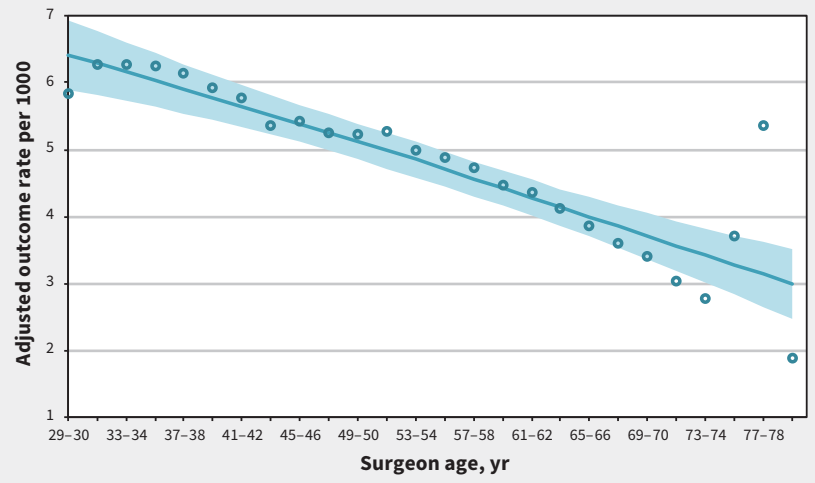

Figure 1: Adjusted outcome rate per 1000 events of (A) composite outcome (comprising death, readmission or complication within $30 \mathrm{~d}$ ), (B) mortality, (C) readmission and (D) complication by surgeon age (yr). We used locally weighted scatterplot smoothing to generate a line of best fit (solid line), with the shaded area representing the $95 \%$ confidence interval. 
(adjusted OR 0.93, 95\% Cl 0.88-0.97) compared with those treated by younger surgeons ( $<65 \mathrm{yr}$ ). This translates to absolute unadjusted differences in event rate (per 1000 patients) for the composite primary outcome, mortality, readmissions and complications between younger surgeons and older surgeons of 31.3 events (95\% $\mathrm{Cl} 31.3-$ 31.4), 5.82 events ( $95 \% \mathrm{Cl} 5.8-5.9), 7.88$ events (95\% Cl 7.8-7.9) and 23.7 events (95\% Cl 23.6-23.7), respectively. However, we did not observe a significant difference in LOS by surgeon age (adjusted RR $1.02,95 \% \mathrm{Cl}$ 0.99-1.04).

We performed stratified analyses by surgical specialty, sex of the surgeon, annual surgical volume of the index procedure, hospital status (academic or community), sex of the patient, patient age and patient comorbidity (Figure 2). We observed differences in the effect of surgeon age on the composite outcome by surgical specialty $\left(p_{\text {interaction }}<0.001\right)$, with a larger benefit among older surgeons observed among plastic (adjusted OR $0.51,95 \% \mathrm{Cl} 0.43-0.61$ ) and vascular (adjusted OR 0.47 , $95 \% \mathrm{Cl} 0.36-0.62$ ) surgeons. We found that younger surgeons had improved outcomes in the specialties of obstetrics and gynecology (adjusted OR 1.02, 95\% Cl 1.01-1.03) and urology (adjusted OR 1.05, 95\% Cl 1.02-1.08). There was no significant interaction between surgeon sex and the composite outcome $\left(p_{\text {interaction }}=0.2\right)$. The effect of surgeon age was consistent across quartiles of annual surgical volume.

Table 2: Adjusted odds ratios for 30-day postoperative outcomes, by covariate

\begin{tabular}{|c|c|c|c|c|c|}
\hline \multirow[b]{2}{*}{ Covariate } & \multicolumn{5}{|c|}{ Adjusted OR $(95 \% \mathrm{Cl})^{\star}$} \\
\hline & $\begin{array}{l}\text { Composite } \\
\text { outcome† }\end{array}$ & Death & $\begin{array}{l}\text { Hospital } \\
\text { readmission }\end{array}$ & Complication & LOS \\
\hline \multicolumn{6}{|l|}{ Surgeon characteristic } \\
\hline $\begin{array}{l}\text { Surgeon age } \\
\text { (increments of } 10 \mathrm{yr} \text { ) }\end{array}$ & $0.95(0.92-0.98)$ & $0.95(0.91-1.00)$ & $0.98(0.97-0.99)$ & $0.94(0.89-0.98)$ & $1.01(0.99-1.02)$ \\
\hline \multicolumn{6}{|l|}{ Surgeon sex } \\
\hline Female v. male & $0.96(0.93-0.99)$ & $0.85(0.73-0.99)$ & $1.00(0.98-1.02)$ & $0.93(0.89-0.98)$ & $0.98(0.94-1.02)$ \\
\hline \multicolumn{6}{|c|}{ Surgeon volume (quartiles) } \\
\hline 1 (lowest) & Ref. & Ref. & Ref. & Ref. & Ref. \\
\hline 2 & $0.92(0.89-0.94)$ & $0.83(0.68-1.01)$ & $0.96(0.94-0.98)$ & $0.91(0.87-0.95)$ & $0.91(0.86-0.97)$ \\
\hline 3 & $0.91(0.87-0.95)$ & $0.67(0.51-0.87)$ & $0.95(0.91-0.99)$ & $0.91(0.81-1.01)$ & $0.88(0.81-0.95)$ \\
\hline 4 (highest) & $0.88(0.84-0.93)$ & $0.66(0.51-0.84)$ & $0.93(0.89-0.97)$ & $0.87(0.79-0.97)$ & $0.85(0.77-0.94)$ \\
\hline \multicolumn{6}{|l|}{ Patient characteristic } \\
\hline \multicolumn{6}{|l|}{ Patient sex } \\
\hline Female v. male & $0.78(0.69-0.88)$ & $0.60(0.40-0.91)$ & $0.92(0.87-0.98)$ & $0.73(0.65-0.82)$ & $0.93(0.85-1.00)$ \\
\hline Patient age & $1.02(1.01-1.02)$ & $1.09(1.06-1.11)$ & $1.01(1.00-1.01)$ & $1.02(1.01-1.02)$ & $1.01(1.01-1.02)$ \\
\hline \multicolumn{6}{|l|}{ Income quintile } \\
\hline 1 (lowest) & Ref. & Ref. & Ref. & Ref. & Ref. \\
\hline 2 & $0.95(0.94-0.96)$ & $0.89(0.83-0.97)$ & $0.95(0.94-0.96)$ & $0.97(0.96-0.99)$ & $0.94(0.92-0.96)$ \\
\hline 3 & $0.92(0.90-0.95)$ & $0.83(0.73-0.95)$ & $0.93(0.91-0.94)$ & $0.94(0.90-0.98)$ & $0.90(0.87-0.93)$ \\
\hline 4 & $0.91(0.88-0.94)$ & $0.78(0.71-0.87)$ & $0.91(0.90-0.93)$ & $0.93(0.88-0.97)$ & $0.88(0.85-0.91)$ \\
\hline 5 (highest) & $0.89(0.85-0.92)$ & $0.73(0.62-0.87)$ & $0.90(0.88-0.92)$ & $0.90(0.85-0.95)$ & $0.86(0.82-0.90)$ \\
\hline \multicolumn{6}{|l|}{ Rurality } \\
\hline Yes v. no & $0.98(0.96-1.00)$ & $1.04(0.93-1.15)$ & $1.04(1.02-1.06)$ & $0.92(0.90-0.94)$ & $1.02(0.97-1.09)$ \\
\hline \multicolumn{6}{|c|}{ Aggregated Diagnosis Group } \\
\hline $0-5$ & Ref. & Ref. & Ref. & Ref. & Ref. \\
\hline $6-7$ & $1.07(1.05-1.09)$ & $0.91(0.81-1.01)$ & $1.11(1.09-1.13)$ & $1.04(1.02-1.05)$ & $1.04(1.01-1.08)$ \\
\hline $8-10$ & $1.21(1.17-1.25)$ & $1.12(0.97-1.30)$ & $1.29(1.25-1.32)$ & $1.11(1.07-1.14)$ & $1.15(1.09-1.21)$ \\
\hline $11+$ & $1.59(1.50-1.69)$ & $1.81(1.44-2.28)$ & $1.68(1.59-1.78)$ & $1.35(1.26-1.45)$ & $1.45(1.30-1.62)$ \\
\hline \multicolumn{6}{|l|}{ Hospital type } \\
\hline Academic v. community & $1.08(1.04-1.11)$ & $1.05(0.86-1.28)$ & $1.06(1.03-1.10)$ & $1.12(1.06-1.19)$ & $0.97(0.86-1.08)$ \\
\hline
\end{tabular}


We performed stratified analyses by emergent and elective procedures to minimize potential bias introduced by patients or referring physicians preferentially selecting surgeons of a specific age group. Older surgeon age was associated with lower odds of the composite outcomes of death, readmission or complication for both emergent (adjusted OR $0.97,95 \% \mathrm{CI}$ 0.94-0.99) and elective (adjusted OR 0.96, 95\% Cl 0.93-0.99) procedures $\left(p_{\text {interaction }}=0.3\right)$ (Table 3$)$. We performed post hoc analyses stratified by case complexity (we defined lowcomplexity cases as those with little variability and performed by a substantial proportion of surgeons within a subspecialty such as appendectomy or cholecystectomy) and era of surgery
(2007-2010 v. 2013-2015). Results were consistent in both strata for these analyses (Appendix 2).

\section{Interpretation}

In this population-based cohort in Ontario, after adjusting for potential cofounders, patients treated by older surgeons had lower rates of 30-day mortality, readmission or complication compared with those treated by younger surgeons, regardless of how we operationalized surgeon age. This applied to both emergent and elective procedures, which reduces the likelihood that the observed association might be explained by patient or surgeon choice.
Specialty

Cardiovascular surgery

General surgery

Neurosurgery

Obstetrics and gynecology

Orthopedic surgery

Otolaryngology

Plastic surgery

Thoracic surgery

Urology

Vascular surgery

Surgeon sex

Female

Male

Surgeon volume, quartiles

First (lowest)

Second

Third

Fourth (highest)

Hospital status

Academic

Community

Patient sex

Female

Male

Patient age, yr

18-35

36-64

$\geq 65$

Patient comorbidity, ADG

0-5

6-7

8-10

$\geq 11$

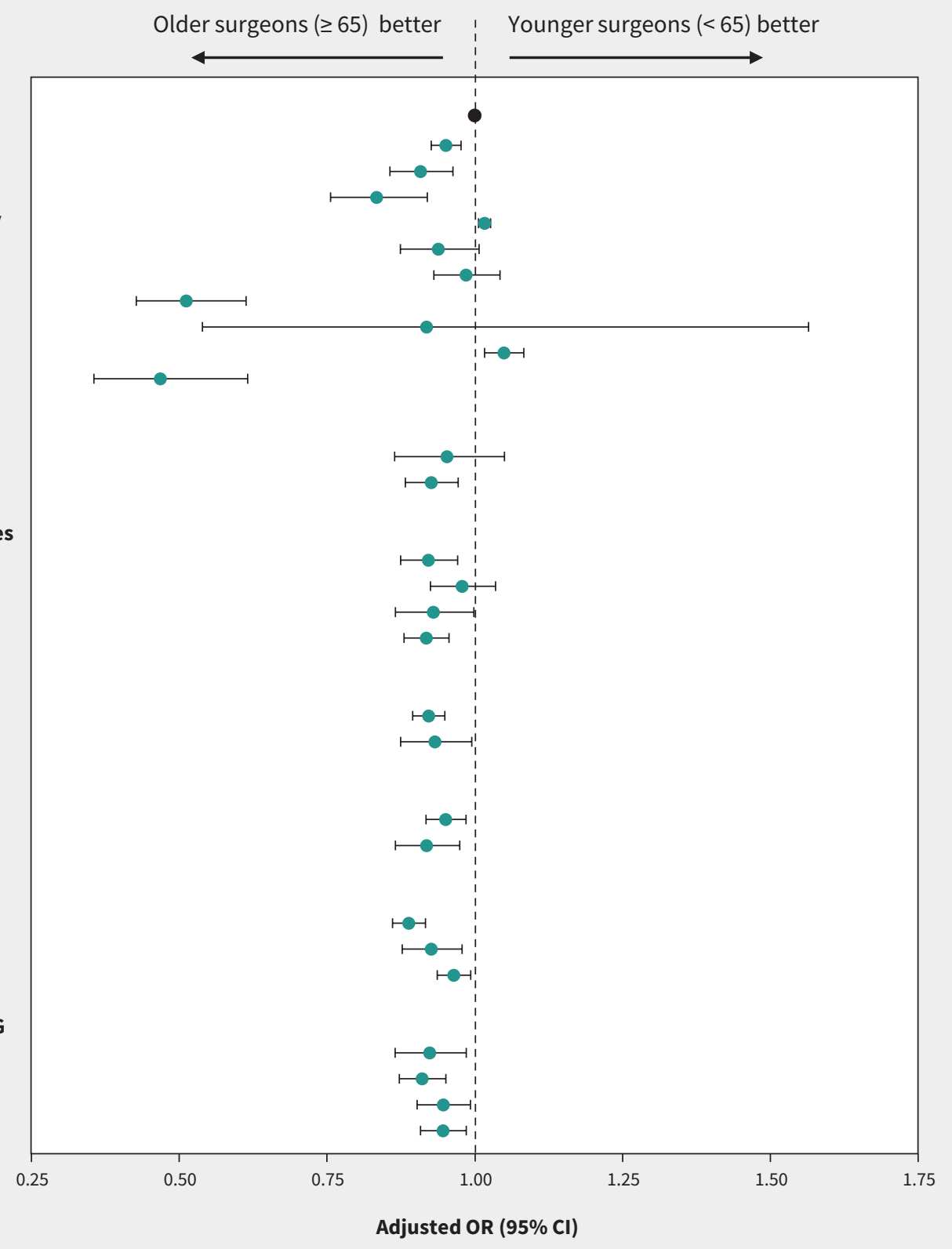

Figure 2: Adjusted odds ratios (ORs) for adverse postoperative outcomes (death, readmission or complications) among patients treated by older ( $\geq 65 \mathrm{yr}$ ) and younger $(<65 \mathrm{yr}$ ) surgeons, stratified by physician, patient and hospital factors in a dichotomized analysis. Note: ADG = Aggregated Diagnosis Group, $\mathrm{Cl}=$ confidence interval. 


\begin{tabular}{|c|c|c|c|}
\hline \multirow[b]{2}{*}{ Outcome } & \multicolumn{2}{|c|}{ OR $(95 \% \mathrm{CI})$} & \multirow{2}{*}{$\begin{array}{l}p \text { value for } \\
\text { interaction }\end{array}$} \\
\hline & Emergent surgery & Elective surgery & \\
\hline $\begin{array}{l}\text { Primary composite outcome (death, } \\
\text { readmission or complication within } 30 \mathrm{~d} \text { ) }\end{array}$ & $0.97(0.94-0.99)$ & $0.96(0.93-0.99)$ & 0.3 \\
\hline Death within $30 \mathrm{~d}$ & $0.98(0.96-1.01)$ & $0.96(0.91-1.01)$ & 0.25 \\
\hline Readmission within $30 \mathrm{~d}$ & $1.00(0.99-1.02)$ & $0.98(0.97-0.99)$ & 0.002 \\
\hline Complication within $30 \mathrm{~d}$ & $0.94(0.91-0.98)$ & $0.94(0.89-0.999)$ & 0.97 \\
\hline Ratio of means for hospital LOS & $1.01(1.01-1.02)$ & Did not converge & \\
\hline \multicolumn{4}{|c|}{$\begin{array}{l}\text { Note: } A D G=\text { Aggregated Diagnosis Group, } \mathrm{Cl} \text { = confidence interval, } \mathrm{LOS}=\text { length of stay, } \mathrm{OR}=\text { odds ratio. } \\
\text { *Younger than } 65 \text { years versus } 65 \text { years and older. } \\
\text { tWe adjusted for patient sex, age, income quintile, geographic region of residence, rurality and comorbidity (ADG score); index } \\
\text { operative vear: surgeon sex: } \text { surgeon specialty: and hospital type (academicy community). }\end{array}$} \\
\hline
\end{tabular}

Our findings likely reflect a combination of changes in the surgeon cohort and surgical practice with age. Surgeons who retire at a younger age may do so as a result of declining capability based on self-, peer- or regulator-based evaluation, or for unrelated reasons. The older surgeons who continue to operate are therefore a highly selected group (4.7\% of surgeons are over $65 \mathrm{yr}$ of age) performing $6 \%$ of the procedures studied. We adjusted for some patient characteristics; however, older surgeons may be able to better select patients at lower risk of postoperative complications, leading to the observed improved outcomes (self-selection).

Our work is consistent with the findings of previous studies that showed better performance by older surgeons, ${ }^{16}$ but we studied both elective and emergent operative procedures over a much wider patient age range than previously studied. Furthermore, we have shown consistency in results by using additional indicators of surgical outcomes beyond mortality alone (i.e., readmission and complications). Our findings differ from those of earlier studies showing worse outcomes in patients treated by older surgeons, both in Medicare beneficiaries and retrospective institutional cohorts. This may reflect procedure specific differences such as for carotid endartectomy, ${ }^{13}$ inguinal hernia repair ${ }^{15}$ or pancreatectomy, ${ }^{13}$ which we did not evaluate or may reflect changes over time.

Because we included a variety of surgical specialties and both elective and emergent procedures, our findings are generalizable across the spectrum of surgical practice. The single-payer health care system in Ontario for surgical procedures means virtually all procedures were captured in our analysis. The use of administrative data allows the comprehensive identification of readmissions or complications after surgery that occurred anywhere in the province.

Our findings argue against mandatory retirement age policies for surgeons and support individualized assessment strategies.,29 Mechanisms may be considered to encourage older surgeons, who remain capable of delivering high-quality care, to remain in practice longer to meet impending shortages in the surgical workforce. ${ }^{30}$ Further research is needed to understand the higher rates of adverse postoperative outcomes in younger surgeons and whether interventions to lower these rates could be started during training, at the time of the credentialing process ${ }^{31}$ or with monitoring and mentoring during early independent practice. ${ }^{32,33}$

\section{Limitations}

Limitations of our study include an inability to account for residual confounding owing to the observational nature of our study. Although we attempted to control for patient comorbidity, case complexity is difficult to measure and our results could be explained by older surgeons operating on less complex patients or younger surgeons receiving disproportionately higher referrals of complex cases. However, a stratified analysis by case complexity as we measured it did not show heterogeneity of effect.

Newer technologies such as robotic-assisted surgery were not widely available in Ontario during the study period and were excluded. The effect of surgeon age for these procedures is unclear; however, we found no heterogeneity of the association between surgeon age and outcomes comparing different years of surgery that may differ in the technologies used. Finally, in addition to generalized estimating equation models (clustered on procedure) we attempted hierarchical modelling for these data at 2 or more levels (e.g., clustering by surgeon and institution), but these models could not be fitted because of computational constraints.

\section{Conclusion}

We found that rates of patient death, readmission to hospital and complications fell in Ontario as surgeon age increased. Further understanding of the mechanisms underlying this finding may be useful in improving patient safety and outcomes.

\section{References}

1. Thomas WE. Teaching and assessing surgical competence. Ann R Coll Surg Engl 2006;88:429-32.

2. Schenarts PJ, Cemaj S. The aging surgeon: implications for the workforce, the surgeon, and the patient. Surg Clin North Am 2016;96:129-38.

3. Dellinger EP, Pellegrini CA, Gallagher TH. The aging physician and the medical profession: a review. JAMA Surg 2017;152:967-71.

4. Eva KW. The aging physician: changes in cognitive processing and their impact on medical practice. Acad Med 2002;77(Suppl):S1-6. 
5. Birkmeyer JD, Stukel TA, Siewers AE, et al. Surgeon volume and operative mortality in the United States. N Engl J Med 2003;349:2117-27.

6. Vickers AJ, Savage CJ, Hruza M, et al. The surgical learning curve for laparoscopic radical prostatectomy: a retrospective cohort study. Lancet Oncol 2009;10:475-80.

7. Schmidt CM, Turrini O, Parikh P, et al. Effect of hospital volume, surgeon experience, and surgeon volume on patient outcomes after pancreaticoduodenectomy: a single-institution experience. Arch Surg 2010;145:634-40.

8. Billingsley KG, Morris AM, Green P, et al. Does surgeon case volume influence nonfatal adverse outcomes after rectal cancer resection? J Am Coll Surg 2008; 206:1167-77.

9. Choudhry NK, Fletcher RH, Soumerai SB. Systematic review: the relationship between clinical experience and quality of health care. Ann Intern Med 2005;142: 260-73.

10. Anderson BR, Wallace AS, Hill KD, et al. Association of surgeon age and experience with congenital heart surgery outcomes. Circ Cardiovasc Qual Outcomes 2017; 10:e003533. doi: 10.1161/CIRCOUTCOMES.117.003533.

11. Bridgewater B, Grayson AD, Au J, et al. Improving mortality of coronary surgery over first four years of independent practice: retrospective examination of prospectively collected data from 15 surgeons. BMJ 2004;329:421.

12. Waljee JF, Greenfield LJ, Dimick JB, et al. Surgeon age and operative mortality in the United States. Ann Surg 2006;244:353-62.

13. O'Neill L, Lanska DJ, Hartz A. Surgeon characteristics associated with mortality and morbidity following carotid endarterectomy. Neurology 2000;55:773-81.

14. Hartz AJ, Kuhn EM, Pulido J. Prestige of training programs and experience of bypass surgeons as factors in adjusted patient mortality rates. Med Care 1999;37:93-103.

15. Neumayer LA, Gawande AA, Wang J, et al.; CSP \#456 Investigators. Proficiency of surgeons in inguinal hernia repair: effect of experience and age. Ann Surg 2005;242:344-8, discussion 348-52.

16. Tsugawa $Y$, Jena AB, Orav EJ, et al. Age and sex of surgeons and mortality of older surgical patients: observational study. BMJ 2018;361:k1343.

17. Wallis CJ, Ravi B, Coburn N, et al. Comparison of postoperative outcomes among patients treated by male and female surgeons: a population based matched cohort study. BMJ 2017;359:j4366.

18. von Elm E, Altman DG, Egger M, et al.; STROBE Initiative. The Strengthening the Reporting of Observational Studies in Epidemiology (STROBE) statement: guidelines for reporting observational studies. Ann Intern Med 2007;147:573-7.

19. Benchimol El, Smeeth L, Guttmann A, et al.; RECORD Working Committee. The REporting of studies Conducted using Observational Routinely-collected health Data (RECORD) statement. PLoS Med 2015;12:e1001885.

20. Williams JI, Young W. Appendix 1: A summary of studies on the quality of health care administrative databases in Canada. In: Goel V, Williams JI, Anderson GM, al., editors. Patterns of Health Care in Ontario: The ICES Practice Atlas. 2nd Edition. Ottawa: Canadian Medical Association; 1996:339-45.

21. Juurlink DN, Preyra C, Croxford R. al. Canadian Institute for Health Information Discharge Abstract Database: a validation study. Toronto: ICES; 2006.

22. Iron K, Zagorski BM, Sykora K, et al. Living and dying in Ontario: an opportunity for improved health information - ICES investigative report. Toronto: ICES Investigative Report; 2008.

23. Govindarajan A, Urbach DR, Kumar M, et al. Outcomes of daytime procedures performed by attending surgeons after night work. N Engl J Med 2015;373:845-53.

24. Urbach DR, Govindarajan A, Saskin R, et al. Introduction of surgical safety checklists in Ontario, Canada. N Engl J Med 2014;370:1029-38.

25. Poushay HM, Kagedan DJ, Hallet J, et al. Why do general surgeons decide to retire?: A population-level survey. Ann Surg 2018;267:e4-5.

26. Local HIN. (LHINs) plan, integrate and fund local health care, improving access and patient experience [main page]. Toronto: Queen's Printer for Ontario; 2014. Available: www.lhins.on.ca/ (accessed 2020 Mar. 11).

27. The Johns Hopkins ACG Case-Mix System reference manual version 7.0. Baltimore: Johns Hopkins Bloomberg School of Public Health; 2005.

28. Austin PC. Using the standardized difference to compare the prevalence of a binary variable between two groups in observational research. Commun Stat Simul Comput 2009;38:1228-34. doi: 10.1080/03610910902859574.

29. Soonsawat A, Tanaka G, Lammando MA, et al. Cognitively impaired physicians: How do we detect them? How do we assist them? Am J Geriatr Psychiatry 2018; 26:631-40.

30. 2018 update: the complexities of physician supply and demand - projections from 2016 to 2030. Washington (DC): HIS Markit Ltd.; 2018.

31. Sachdeva AK, Flynn TC, Brigham TP, et al.; American College of Surgeons (ACS) Division of Education; Accreditation Council for Graduate Medical Education (ACGME). Interventions to address challenges associated with the transition from residency training to independent surgical practice. Surgery 2014;155:867-82.

32. Wakeam E, Feinberg S. Surgeon unemployment: would practice sharing be a viable solution? Can J Surg 2016;59:141-2.

33. Cogbill TH, Shapiro SB. Transition from training to surgical practice. Surg Clin North Am 2016;96:25-33.

which permits others to distribute, remix, adapt and build upon this work, for commercial use, provided the original work is properly cited. See: http://creativecommons.org/licenses/by/4.0/.

Data sharing: Data are protected by agreements between the Ontario Ministry of Health and Long-Term Care and ICES, which preclude the authors from sharing the source data.

Acknowledgements: The authors are indebted to the methodologic and analytic support provided by Refik Saskin, Lisa Ellison and Bo Zhang who were compensated for their work.

Disclaimer: This study made use of de-identified data from the ICES Data Repository, which is managed by the ICES with support from its funders and partners: Canada's Strategy for Patient-Oriented Research (SPOR), the Ontario SPOR Support Unit, the Canadian Institutes of Health Research and the Government of Ontario. The opinions, results and conclusions reported in this paper are those of the authors and are independent from the funding sources. No endorsement by ICES, which is funded by an annual grant from the Ontario Ministry of Health and Long-Term Care (MOHLTC), or MOHLTC is intended or should be inferred. We used data and information compiled and provided by the Canadian Institute for Health Information (CIHI). However, the analyses, conclusions, opinions and statements expressed herein are those of the authors, and not necessarily those of $\mathrm{CIHI}$.

Accepted: Feb. 10, 2020

Correspondence to: Raj Satkunasivam, raj.satkunasivam@gmail.com 